\title{
Erratum to: Optimal Axisymmetric Noses of Bodies in a Flow. Calculations and Experiments
}

\author{
I. P. Bol'shiyanov ${ }^{1 *}$, N. N. Zakharov ${ }^{1,2 * *}$, K. S. P'yankov ${ }^{2 * * *}$, and N. I. Tillyaeva $2^{* * * *}$ \\ ${ }^{I}$ Institute of Applied Mechanics of Russian Academy of Sciences \\ Leningradski pr. 7, Moscow, 125040 Russia \\ ${ }^{2}$ Baranov Central Institute of Aviation Motors (CIAM) \\ ul. Aviamotornaya 2, Moscow, 111116 Russia \\ Received January 30, 2019; in final form, January 30, 2019
}

DOI: $10.1134 / \mathrm{S} 0015462818070017$

Page 303, last line should read: (project No. 17-01-00126).

The original article can be found online at https://doi.org/10.1134/S0015462818020052.

\footnotetext{
${ }^{*}$ E-mail: paleo88@mail.ru.

${ }^{* *}$ E-mail: zakharov@ciam.ru .

${ }^{* * *}$ E-mail: kirill@ciam.ru.

${ }^{* * * *}$ E-mail: ntill@ciam.ru.
} 